\title{
Article \\ Correlation between Commercial Anti-RBD IgG Titer and Neutralization Titer against SARS-CoV-2 Beta Variant
}

\author{
Rosana Wing-Shan Poon ${ }^{1,2}$, Lu Lu ${ }^{2}$, Carol Ho-Yan Fong ${ }^{2}$, Tak-Chuen Ip ${ }^{1}$, Lin-Lei Chen ${ }^{2}$, Ricky Rui-Qi Zhang ${ }^{2}{ }^{\oplus}$, \\ Cyril Chik-Yan Yip ${ }^{1,2}$, Vincent Chi-Chung Cheng ${ }^{1,2}$, Kwok-Hung Chan ${ }^{2}{ }^{\mathbb{D}}$, Kwok-Yung Yuen ${ }^{1,2,3}$ \\ and Kelvin Kai-Wang To ${ }^{1,2,3, *}$
}

check for updates

Citation: Poon, R.W.-S.; Lu, L.; Fong, C.H.-Y.; Ip, T.-C.; Chen, L.-L.; Zhang, R.R.-Q.; Yip, C.C.-Y.; Cheng, V.C.-C.; Chan, K.-H.; Yuen, K.-Y.; et al. Correlation between Commercial Anti-RBD IgG Titer and Neutralization Titer against SARS-CoV-2 Beta Variant. Diagnostics 2021, 11, 2216. https://doi.org/ $10.3390 /$ diagnostics11122216

Academic Editor: Anna Baraniak

Received: 6 October 2021

Accepted: 24 November 2021

Published: 27 November 2021

Publisher's Note: MDPI stays neutral with regard to jurisdictional claims in published maps and institutional affiliations.

Copyright: (c) 2021 by the authors. Licensee MDPI, Basel, Switzerland. This article is an open access article distributed under the terms and conditions of the Creative Commons Attribution (CC BY) license (https:// creativecommons.org/licenses/by/ $4.0 /)$.
1 Department of Microbiology, Queen Mary Hospital, Hong Kong, China; rosana@hku.hk (R.W.-S.P.); chuen1220@gmail.com (T.-C.I.); cyrilyip2@gmail.com (C.C.-Y.Y.); vccheng@hku.hk (V.C.-C.C.); kyyuen@hku.hk (K.-Y.Y.)

2 Department of Microbiology, Li Ka Shing Faculty of Medicine, The University of Hong Kong, Hong Kong, China; u3003963@connect.hku.hk (L.L.); carolfong.hy@gmail.com (C.H.-Y.F.); chenlinlei2346@gmail.com (L.-L.C.); zhangrq@hku.hk (R.R.-Q.Z.); chankh2@hku.hk (K.-H.C.)

3 State Key Laboratory for Emerging Infectious Diseases, Carol Yu Centre for Infection, Department of Microbiology, Li Ka Shing Faculty of Medicine, The University of Hong Kong, Pokfulam, Hong Kong, China

* Correspondence: kelvinto@hku.hk; Tel.: +852-22552413; Fax: +852-28551241

Abstract: Objectives: The emergence of SARS-CoV-2 variants of concern (VOCs) have diminished the effectiveness of vaccines and are associated with a rebound in the number of COVID-19 cases globally. These variants contain mutations at the spike (S) protein receptor binding site (RBD), which affect antibody binding. Current commercially available antibody assays were developed before the VOCs emerged. It is unclear whether the levels of these commercially available antibody assays can predict the neutralizing antibody titers against the VOCs. In this study, we sought to determine the correlation between the binding antibody concentration and microneutralization antibody titer against the beta variant. Methods: This study included 58 COVID-19 patients. The concentrations of IgG against the SARS-CoV-2 spike protein RBD and nucleocapsid (N) protein were measured using the Abbott SARS-CoV-2 IgG II Quant assay and the SARS-CoV-2 IgG assay, respectively. The neutralization antibody titer against the wild type lineage A SARS-CoV-2 and against the beta variant (B.1.351) was determined using a conventional live virus neutralization test. Results: The geometric mean MN titer (GMT) against the beta variant was significantly lower than that against the wild type lineage A virus (5.6 vs. 47.3, $p<0.0001$ ). The anti-RBD IgG had a better correlation with the neutralizing antibody titer than that of the anti-N IgG assay against the wild type lineage A virus (Spearman rho, 0.5901 vs. 0.3827). However, the correlation between the anti-RBD or the anti-N IgG and the MN titer against the beta variant was poor. Conclusions: Currently available commercial antibody assays may not predict the level of neutralizing antibodies against the variants. A new generation of antibody tests specific for variants are required.

Keywords: COVID-19; SARS-CoV-2; antibody; receptor binding domain; neutralization

\section{Introduction}

The COVID-19 pandemic has constituted a major threat to humans. Serological assays for SARS-CoV-2 have played important roles in clinical management, outbreak control, and serosurveillance studies [1,2]. For diagnostics, antibody tests are especially useful for patients whose molecular test results are inconclusive, if the patient presents late and the virus is already cleared, or if the patient has a suspected reinfection [3,4]. Seroprevalence studies are pivotal in uncovering the true burden of infection, as infections confirmed by RT-PCR only represent the tip of the iceberg [2]. Serological assays are also critical for evaluating the immunogenicity of vaccines, and this is especially important with the emergence of variants. During contact tracing, the combination of epidemiological and 
antibody test information can help to identify the source of an outbreak [4]. Antibody tests have helped to guide decisions on patient discharge, especially for patients with prolonged viral shedding.

There are three major types of serological assays for COVID-19 [1,5]. Antibodies against a specific SARS-CoV-2 protein, such as the N protein or the S protein, can be detected by a chemiluminescence immunoassay (CLIA), an enzyme immunoassay assay (EIA), or a lateral flow assay (LFA) [6]. Neutralizing antibodies can be measured by a conventional live virus neutralization test (cVNT), pseudovirus neutralization assay, and surrogate virus neutralization assay (sVNT) $[7,8]$. Although cVNT is the "gold standard" serological assay for diagnosis and determination of protective immunity, it can only be performed in biosafety level 3 laboratories and is time consuming. On the other hand, CLIA, EIA, LFA, or sVNT are technically easy and can be performed in biosafety level 2 laboratories, and these assays are available commercially.

Since late 2019, several SARS-CoV-2 variants with RBD mutations have emerged. The World Health Organization has classified the alpha (B.1.1.7), beta (B.1.351), gamma (P.1), and delta (B.1.617.2) variants as VOCs [9]. The beta, gamma, and delta variants are more resistant to neutralization by the convalescent serum collected from COVID-19 vaccine recipients or from patients naturally infected by SARS-CoV-2 without RBD mutations [10]. Since current commercial antibody assays were designed based on the wild type SARS-CoV-2, we hypothesized that the antibody concentration determined by these commercial assays may not correlate well with the neutralizing antibody titer. In this study, we sought to determine the correlation between the binding antibody concentration and microneutralization antibody titer against the beta variant.

\section{Materials and Methods}

\subsection{Patient Specimens}

A total of 58 archived serum specimens from COVID-19 patients were retrieved from the Clinical Microbiology Laboratory of Queen Mary Hospital. These specimens were collected between February and May 2020 at a median of 36 days post symptom onset (IQR: 23-46 days). These patients had not received a COVID-19 vaccine before the collection of the serum specimens. This study was approved by the Institutional Review Board of the University of Hong Kong/Hospital Authority Hong Kong West Cluster (UW 13-372). Written informed consent was waived as only archived specimens were used.

\subsection{Chemiluminescent Microparticle Immunoassay for the Detection of Anti-N and Anti-RBD IgG}

We tested specimens for anti-N IgG using the Abbott SARS-CoV-2 IgG assay and for anti-RBD IgG using the SARS-CoV-2 IgG II Quant assay according to the manufacturer's instructions. Both assays, based on the CLIA platform, were performed on the Architect i2000 SR immunoassay analyzer (Abbott Diagnostics, Chicago, IL, USA).

The manufacturers' recommended cut-off for seropositivity was a S/CO value of 1.4 for the anti-N IgG qualitative assay and $50 \mathrm{AU} / \mathrm{mL}$ for the anti-RBD IgG quantitative assay.

\subsection{Conventional Live Virus Neutralization Assay}

A cVNT assay was performed as we described previously [10,11]. The virus isolates included the SARS-CoV-2 HKU-001a (wild type lineage A; GenBank accession number MT230904) [12] and the beta variant (Lineage B.1.351; GISAID accession number: EPI_ISL_2423556). The microneutralization (MN) antibody titer was the highest dilution with a $50 \%$ inhibition of cytopathic effect. An MN titer of $\geq 10$ was considered positive. A viral culture of SARS-CoV-2 and cVNT assays were conducted in a biosafety level 3 facility.

\subsection{Statistical Analysis}

A statistical analysis was performed using a GraphPad PRISM 9.2.0 (GraphPad Software). For the purpose of statistical calculation, an MN titer of $<10$ was assigned a value 
of 5. A Wilcoxon matched-pairs signed rank test was used for comparing the MN titers against the wild type lineage A and lineage B.1.351 virus. The correlation between binding the antibody concentration (anti-RBD IgG or anti-N IgG) and the MN titer was determined using Spearman's correlation. A $p$ value of $<0.05$ was considered statistically significant.

\section{Results}

The GMT against the wild type lineage A virus (GMT: 47.3; range: 10-320) was significantly higher than that against the beta variant (GMT: 5.6; range $<10-20)(p<0.0001)$ (Figure 1A). There was a statistically significant degree of correlation between the antiRBD IgG and the anti-N IgG with a Spearman rho of 0.4666 ( $95 \%$ confidence interval [C.I. 0.2294-0.6514]; $p=0.0002$ ) (Figure 1B).

There was a statistically significant correlation between the concentrations of the anti-RBD IgG or the anti-N IgG with the HKU-001a MN titer. The correlation between the anti-RBD IgG concentration and the MN titer (Spearman rho: 0.5901 [95\% C.I. 0.3849-0.7398, $\mathrm{P}<0.0001]$ ) was higher than that between the anti-N IgG S/CO and the MN titer (Spearman rho: 0.3827 [95\% C.I. 0.1303-0.5884, $p=0.0030]$ ) (Figure 1C,D). However, the correlation between the anti-RBD IgG or anti-N IgG with the MN titer against B.1.351 was not statistically significant ( $p=0.8284$ and $p=0.6938$, respectively) (Figure 1E,F).

A

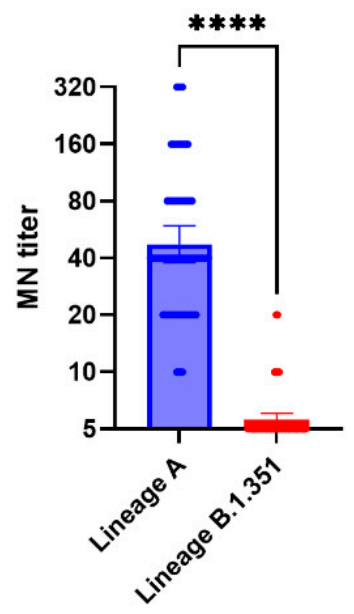

C

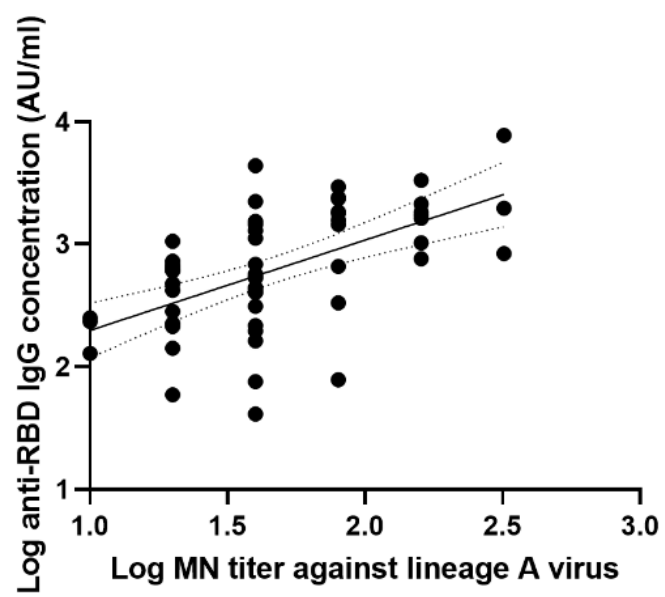

B

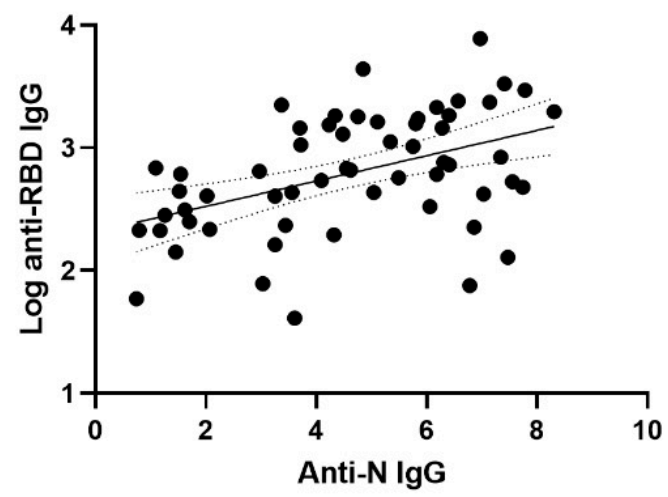

D

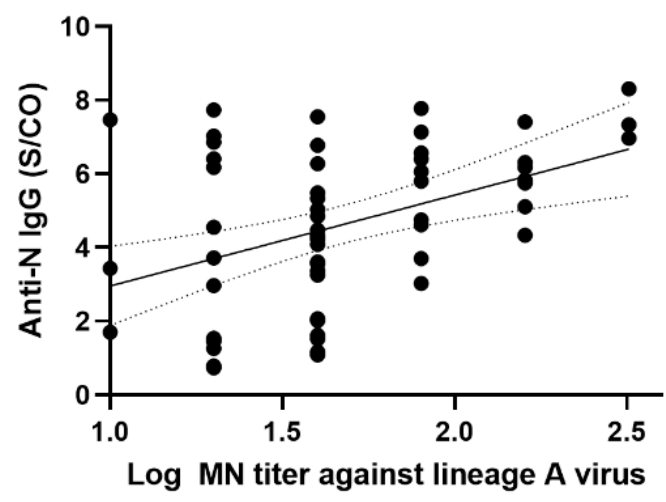

Figure 1. Cont. 
E

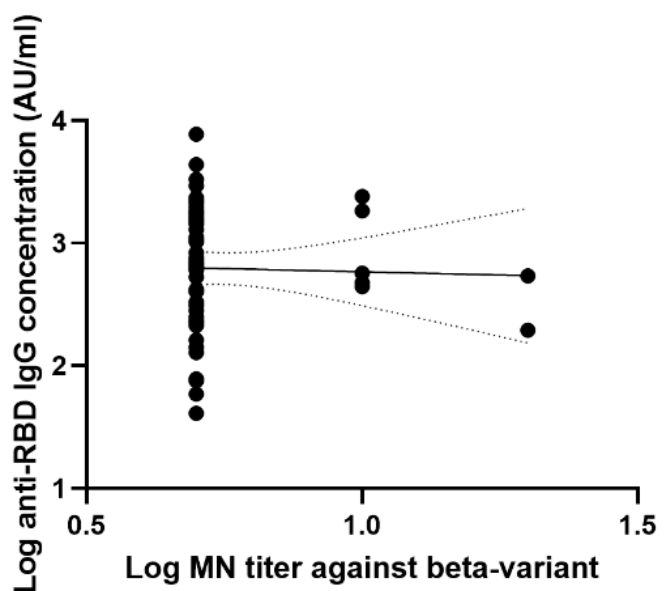

F

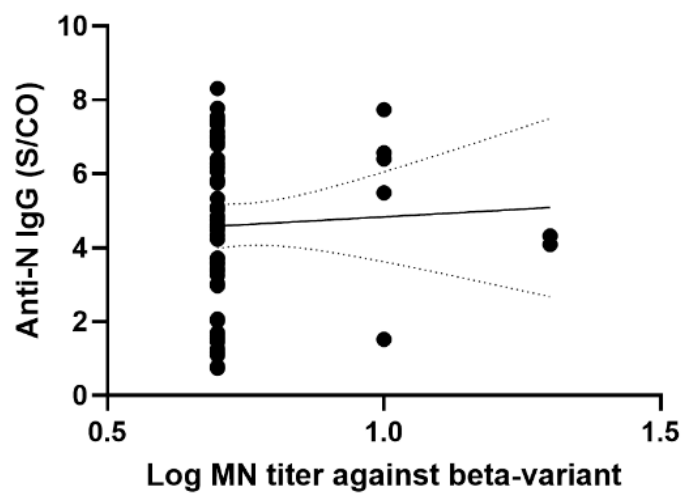

Figure 1. Correlation between MN titer and anti-N or anti-RBD IgG titers. (A) Comparison between MN titers against wild type lineage A virus (HKU-001a) and lineage B.1.351 virus (beta variant). The data represent geometric mean titer and $95 \%$ confidence interval ${ }^{* * *}, p<0.0001$. (B) Correlation between anti-RBD IgG and anti-N IgG. (C,D) Correlation between MN titer against wild type lineage A and anti-RBD IgG (C) or anti-N IgG (D); (E,F) Correlation between MN titer against lineage B.1.351 and anti-RBD IgG (E) or anti-N IgG (F). For (B-F), the solid lines indicate the best fit line, while the dotted lines indicate the $95 \%$ confidence interval.

For specimens with anti-RBD IgG concentrations of $\geq 1000 \mathrm{AU} / \mathrm{mL}, 95.5 \%(21 / 22)$ had an MN titer against HKU-001A of $\geq 40$. However, only $9.1 \%(2 / 22)$ had a beta variant MN titer of 10-20, and none had a beta variant titer of 40 or above (Table 1 ).

Table 1. Correlation between binding antibody titer and microneutralization antibody titer.

\begin{tabular}{|c|c|c|c|c|c|c|}
\hline \multirow[t]{2}{*}{ MN Titer } & \multicolumn{3}{|c|}{ Anti-RBD IgG (AU/mL) } & \multicolumn{3}{|c|}{ Anti-N IgG (S/CO) } \\
\hline & $\begin{array}{c}<50 \\
(n=1)\end{array}$ & $\begin{array}{l}50-999 \\
(n=35)\end{array}$ & $\begin{array}{l}\geq 1000 \\
(n=22)\end{array}$ & $\begin{array}{c}<1.4 \\
(n=5)\end{array}$ & $\begin{array}{l}1.4-4.9 \\
(n=26)\end{array}$ & $\begin{array}{c}\geq 5.0 \\
(n=27)\end{array}$ \\
\hline \multicolumn{7}{|c|}{$\begin{array}{l}\text { Against wild type lineage A } \\
\text { (HKU-001a) }\end{array}$} \\
\hline $10-20$ & $0(0)$ & 15 (42.9) & $1(4.5)$ & $3(60)$ & $7(26.9)$ & $6(22.2)$ \\
\hline$\geq 40$ & $1(100)$ & $20(57.1)$ & $21(95.5)$ & $2(40)$ & 19 (73.1) & $21(77.8)$ \\
\hline \multicolumn{7}{|c|}{$\begin{array}{c}\text { Against beta variant } \\
\text { (B.1.351) }\end{array}$} \\
\hline$<10$ & $1(100)$ & $30(85.7)$ & $20(90.9)$ & $5(100)$ & $23(88.5)$ & $23(85.2)$ \\
\hline 10-20 & $0(0)$ & 5 (14.3) & $2(9.1)$ & $0(0)$ & $3(11.5)$ & 4 (14.8) \\
\hline$\geq 40$ & $0(0)$ & $0(0)$ & $0(0)$ & $0(0)$ & $0(0)$ & $0(0)$ \\
\hline
\end{tabular}

\section{Discussion}

SARS-CoV-2 binding antibody assays are usually used as a surrogate for determining the immune response because it is easy to perform without the need for a biosafety level 3 facility. Studies early during the COVID-19 pandemic showed that the anti-RBD IgG concentration has a high correlation with MN titers [13]. In this study, we confirmed that both the anti-RBD IgG and anti-N IgG concentrations showed a correlation with $\mathrm{MN}$ titers against the wild type lineage A virus. However, the anti-RBD IgG and anti-N IgG concentrations did not correlate with the MN titer against the beta variant. Even for serum with a high anti-RBD IgG $\geq 1000 \mathrm{AU} / \mathrm{mL}, 90.9 \%$ had no detectable MN antibody titer against the beta variant. This is of particular concern as our results suggest that the 
current commercial antibody binding assays that were designed prior to the appearance of RBD variants are not useful in predicting a neutralizing antibody response against some variants.

The beta variant contains the RBD mutations K417N, E484K, and N501Y. Since antiRBD IgG measure all antibodies that bind to the RBD, our results suggest that these anti-RBD IgG do not neutralize the beta variant virus. Studies with recombinant RBD with different mutations showed that E484K alone can significantly affect the binding of serum antibodies to the RBD [10]. E484K has been shown to destabilize the native conformation at the tip of the RBD, which is important for neutralization [14].

There are several limitations in this study. First, we did not include pediatric patients in this study. Weisberg et al. showed the distinct antibody responses in children and adults after a SARS-CoV-2 infection. Adult patients generated antibodies against both the $\mathrm{S}$ and $\mathrm{NP}$ proteins, while children generated antibodies against the $S$ protein [15]. Hence, the results may be different among children. Second, the anti-N assay is not intended to be a quantitative assay. Third, we have not tested the MN titer against other VOCs.

The commercial binding antibody assay, especially the anti-RBD IgG assays, are widely used for the assessment of vaccine immunity, especially in non-research settings. The results from this study suggest that the concentration of anti-RBD IgG as determined by these commercial antibody assays should not be used in predicting the level of neutralizing antibodies against variants. Newer versions of anti-RBD IgG incorporating RBD with different mutations would be required for a better correlation with neutralization antibody titers.

Author Contributions: R.W.-S.P. and K.K.-W.T. designed the study. R.W.-S.P., T.-C.I., C.H.-Y.F., L.-L.C., L.L. and R.R.-Q.Z. acquired the data. K.K.-W.T. carried out the statistical analysis. All authors interpreted the data, revised the manuscript critically for important intellectual content, and approved the final report. All authors have read and agreed to the final version of the manuscript.

Funding: This study is supported by the Consultancy Service for Enhancing Laboratory Surveillance of Emerging Infectious Diseases and Research Capability on Antimicrobial Resistance for the Department of Health of the HKSAR Government, and donations of Richard Yu and Carol Yu, May Tam Mak Mei Yin, the Shaw Foundation Hong Kong, Michael Seak-Kan Tong, the Respiratory Viral Research Foundation Limited, Hui Ming, the Hui Hoy and Chow Sin Lan Charity Fund Limited, the Chan Yin Chuen Memorial Charitable Foundation, Marina Man-Wai Lee, the Jessie \& George Ho Charitable Foundation, Perfect Shape Medical Limited, Kai Chong Tong, and Tse Kam Ming Laurence.

Institutional Review Board Statement: This study was approved by the Institutional Review Board of the University of Hong Kong/Hospital Authority Hong Kong West Cluster (UW 13-372).

Informed Consent Statement: Written informed consent was waived as only archived specimens were used.

Data Availability Statement: The data used to support the findings of this study are included within the article.

Conflicts of Interest: All authors declare no conflict of interest.

\section{References}

1. Ong, D.S.; Fragkou, P.C.; Schweitzer, V.A.; Chemaly, R.F.; Moschopoulos, C.D.; Skevaki, C. How to interpret and use COVID-19 serology and immunology tests. Clin. Microbiol. Infect. 2021, 27, 981-986. [CrossRef] [PubMed]

2. To, K.K.W.; Cheng, V.C.C.; Cai, J.P.; Chan, K.H.; Chen, L.L.; Wong, L.H.; Choi, C.Y.K.; Fong, C.H.Y.; Ng, A.C.K.; Lu, L.; et al. Seroprevalence of SARS-CoV-2 in Hong Kong special administrative region and our returnees evacuated from Hubei province of China: A multi-cohort study. Lancet Microbe 2020, 1, e111-e118. [CrossRef]

3. To, K.K.W.; Hung, I.F.N.; Chan, K.H.; Yuan, S.; To, W.K.; Tsang, D.N.C.; Cheng, V.C.C.; Chen, Z.; Kok, K.H.; Yuen, K.Y. Serum antibody profile of a patient with Coronavirus disease 2019 reinfection. Clin. Infect. Dis. 2021, 72, e659-e662. [CrossRef]

4. To, K.K.W.; Tsang, O.T.Y.; Leung, W.S.; Tam, A.R.; Wu, T.C.; Lung, D.C.; Yip, C.C.Y.; Cai, J.P.; Chan, J.M.C.; Chik, T.S.H.; et al. Temporal profiles of viral load in posterior oropharyngeal saliva samples and serum antibody responses during infection by SARS-CoV-2, An observational cohort study. Lancet Infect. Dis. 2020, 20, 565-574. [CrossRef] 
5. To, K.K.W.; Sridhar, S.; Chiu, K.H.Y.; Hung, D.L.L.; Li, X.; Hung, I.F.N.; Tam, A.R.; Chung, T.W.H.; Chan, J.F.W.; Zhang, A.J.X.; et al. Lessons learned one year after SARS-CoV-2 emergence leading to COVID-19 pandemic. Emerg. Microbes Infect. 2021, 10, 507-535. [CrossRef] [PubMed]

6. Fong, C.H.Y.; Cai, J.P.; Dissanayake, T.K.; Chen, L.L.; Choi, C.Y.K.; Wong, L.H.; Ng, A.C.K.; Pang, P.K.; Ho, D.T.Y.; Poon, R.W.S.; et al. Improved detection of antibodies against SARS-CoV-2 by microsphere-based antibody assay. Int. J. Mol. Sci. 2020, 21, 6595. [CrossRef]

7. Tan, C.W.; Chia, W.N.; Qin, X.; Liu, P.; Chen, M.I.C.; Tiu, C.; Hu, Z.; Chen, V.C.W.; Young, B.E.; Sia, W.R.; et al. A SARS-CoV-2 surrogate virus neutralization test based on antibody-mediated blockage of ace2-spike protein-protein interaction. Nat. Biotechnol. 2020, 38, 1073-1078. [CrossRef] [PubMed]

8. Liu, L.; To, K.K.W.; Chan, K.H.; Wong, Y.C.; Zhou, R.; Kwan, K.Y.; Fong, C.H.Y.; Chen, L.L.; Choi, C.Y.K.; Lu, L.; et al. High neutralizing antibody titer in intensive care unit patients with COVID-19. Emerg. Microbes Infect. 2020, 9, 1664-1670. [CrossRef] [PubMed]

9. Boehm, E.; Kronig, I.; Neher, R.A.; Eckerle, I.; Vetter, P.; Kaiser, L. Novel SARS-CoV-2 variants: The pandemics within the pandemic. Clin. Microbiol. Infect. 2021, 27, 1109-1117. [CrossRef] [PubMed]

10. Chen, L.L.; Lu, L.; Choi, C.Y.K.; Cai, J.P.; Tsoi, H.W.; Chu, A.W.H.; Ip, J.D.; Chan, W.M.; Zhang, R.R.; Zhang, X.; et al. Impact of SARS-CoV-2 variant-associated RBD mutations on the susceptibility to serum antibodies elicited by COVID-19 infection or vaccination. Clin. Infect. Dis. 2021, 26, ciab656. [CrossRef] [PubMed]

11. Lu, L.; Chu, A.W.H.; Zhang, R.R.; Chan, W.M.; Ip, J.D.; Tsoi, H.W.; Chen, L.L.; Cai, J.P.; Lung, D.C.; Tam, A.R.; et al. The impact of spike n501y mutation on neutralizing activity and rbd binding of SARS-CoV-2 convalescent serum. EBioMedicine 2021, $71,103544$. [CrossRef] [PubMed]

12. Chu, H.; Chan, J.F.W.; Yuen, T.T.T.; Shuai, H.; Yuan, S.; Wang, Y.; Hu, B.; Yip, C.C.Y.; Tsang, J.O.L.; Huang, X.; et al. Comparative tropism, replication kinetics, and cell damage profiling of SARS-CoV-2 and SARS-CoV with implications for clinical manifestations, transmissibility, and laboratory studies of COVID-19, An observational study. Lancet Microbe 2020, 1, e14-e23. [CrossRef]

13. GeurtsvanKessel, C.H.; Okba, N.M.; Igloi, Z.; Bogers, S.; Embregts, C.W.; Laksono, B.M.; Leijten, L.; Rokx, C.; Rijnders, B.; Rahamat-Langendoen, J.; et al. An evaluation of COVID-19 serological assays informs future diagnostics and exposure assessment. Nat. Commun. 2020, 11, 3436. [CrossRef] [PubMed]

14. Gobeil, S.; Janowska, K.; McDowell, S.; Mansouri, K.; Parks, R.; Stalls, V.; Kopp, M.F.; Manne, K.; Saunders, K.O.N.; Edwards, R.J.; et al. Effect of natural mutations of SARS-CoV-2 on spike structure, conformation, and antigenicity. Science 2021, 6, eabi6226. [CrossRef]

15. Weisberg, S.P.; Connors, T.J.; Zhu, Y.; Baldwin, M.R.; Lin, W.H.; Wontakal, S.; Szabo, P.A.; Wells, S.B.; Dogra, P.; Gray, J.; et al. Distinct antibody responses to SARS-CoV-2 in children and adults across the COVID-19 clinical spectrum. Nat. Immunol. 2021, 22, 25-31. [CrossRef] 